\title{
A aplicação de técnicas de imposição de mãos no estresse-ansiedade: revisão sistemática da literatura
}

\author{
Pedro Mouráo Roxo da Motta, Nelson Filice de Barros
}

\author{
Laboratório de Práticas Alternativas Complementares e Integrativas em Saúde - Lapacis, \\ Departamento de Saúde Coletiva, Faculdade de Ciências Médicas, Universidade Estadual de \\ Campinas - Unicamp, Campinas, SP, Brasil.
}

\begin{abstract}
Resumo: O presente artigo objetiva analisar, por revisão sistemática da literatura, o uso e o efeito das técnicas de imposição de mãos: Reiki, Toque terapêutico e Toque de cura, no estresse-ansiedade. Foram feitas buscas no PubMed/MedLine, Lilacs, Web of Science, Ebsco e Embase, em outubro de 2013, selecionados 24 artigos e analisados conforme: metodologia,objeto de estudo, fatores de impacto dos jornais, países de desenvolvimento, ano de publicação e resultados alcançados. A literatura mostra: predominância de metodologia quantitativa, 21 dos 24 estudos; uso das técnicas de imposição de mãos em pacientes com sintomas de estresse-ansiedade em tratamento do câncer, em 8 dos 24 estudos; predomínio de publicações na América do Norte, 19 dos 24, sendo 14 deles dos EUA; campo de pesquisa muito novo, com a publicação do primeiro artigo indexado em 1999; publicações em jornais de baixo ou nenhum fator de impacto, apenas 8 dos 24 artigos foram publicados em jornais que apresentaram descrição no Journal Citation Reports; e predominância de estudos estatisticamente significativos, 18 dos 24 artigos. Embora a maioria dos estudos tenha apresentado resultados positivos, são necessários mais estudos para desenvolver a relação do uso de Reiki, Toque terapêutico e Toque de cura para o tratamento de estresse-ansiedade.
\end{abstract}

Palavras-chave: Reiki, Toque Terapêutico, Toque de Cura, Stress, Ansiedade.

\section{The application of the technique of imposition of hands on stress-anxiety: a systematic literature review}

\begin{abstract}
This article aims to analyze, according to a systematic review of the literature, the use and effect of techniques of imposition of hands: Reiki, Therapeutic Touch, and Healing Touch on stress-anxiety. Searches were performed in PubMed/Medline, Lilacs, Web of Science, Embase and Ebsco databases in October 2013; 24 articles were selected and analyzed according to the following issues: methodology, object of study, impact factors of journals, countries where the studies were developed, year of publication, and results. The literature shows predominance of quantitative methodology, 21 of the 24 studies; use of techniques of imposition of hands in patients with symptoms of stress-anxiety in cancer treatment, 8 of the 24 studies; predominance of publications in North America, 19 of the 24 studies, 14 in the U.S.; very new field of research, with the publication of the first indexed article in 1999; publication in journals with low or no impact factor, only 8 of the 24 articles were published in periodicals listed in the Journal Citation Reports; and predominance of statistically significant articles, 18 of 24 studies. Although most studies have shown positive results, the data are insufficient and further studies are needed to develop the relationship of the use of Reiki, Therapeutic Touch, and Healing Touch for the treatment of stress-anxiety.
\end{abstract}

Keywords: Reiki, Therapeutic Touch, Healing Touch, Stress, Anxiety. 


\section{Introdução}

As técnicas de imposição de mãos ou impostação de mãos são Práticas Integrativas e Complementares (PIC) que visam o reequilíbrio e harmonização do sistema energético do ser humano. No campo da ciência, as técnicas de imposição de mãos mais estudadas atualmente são o Reiki, Toque terapêutico (TT) e Toque de cura (TC) (OLIVEIRA, 2003).

O Reiki é uma técnica de canalização de energia vital através de símbolos e sons sagrados, esta energia ainda não é qualificada pela física moderna, porém alguns autores relatam a existência de um biocampo, que seria o Campo de Energia (CE) intrínseco a todos os seres vivos, chamado também de campo quântico, clássico e não clássico (VANDERVAART et al., 2011).

O Reiki é comumente aplicado por meio do toque das mãos no corpo do paciente em posições específicas, ou à distância. A palavra "Rei” em japonês significa universal e "Ki" é o termo usado para descrever a energia vital. Da uniáo da palavra "Rei-universal" com a palavra "Ki-energia vital" temos "energia vital universal". Em uma aplicação de Reiki não existem contraindicaçóes, pois, pelo atributo "Rei" da energia, o "Ki" se direciona para onde é necessário, o que atenua a preocupação do terapeuta em relação à perda de sua energia, pois essa ao passar pelo seu corpo, antes de chegar ao paciente, beneficia também o terapeuta (VANDERVAART et al., 2009).

Segundo a literatura pesquisada, Mikao Usui é o redescobridor do Reiki, porém existe uma diversidade de histórias diferentes contadas, o que se justifica devido às adaptações feitas para introduzir essa prática no mundo ocidental (BABENKO, 2004). Alguns autores citam que a origem do Reiki remonta 3.000 anos a.C. nas escrituras sagradas do Tibet (Sutras Tibetanos) (STEWART, 1995). Porém, até o momento, não existem provas empíricas que sustentem essa afirmação.

No ano de 1925 Mikao Usui iniciou Chujiro Hayashi, médico aposentado da marinha, como Mestre Professor de Reiki, o qual leva essa terapia para sua clínica em Tóquio. Em 1930 Usui morre, e em 1939 Chujiro Hayashi inicia Hawayo Takata como Mestre, Takata, por sua vez, trabalha intensamente o Reiki no Havaí, Japão e EUA, formando 22 Mestres entre homens e mulheres; o ocidente veio conhecer o Reiki em meados de 1970, e o Brasil em 1982 (USUI; PETTER, 1999).

Com a chegada do Reiki no ocidente esta técnica sofreu inúmeras alteraçôes para se adaptar a esta cultura. Na época de Mikao Usui o Reiki era aplicado de maneira mais intuitiva, não existia um tratamento padronizado como atualmente no ocidente, havia, invés disso, um método de diagnóstico onde os alunos treinavam intensamente captar distúrbios energéticos no corpo para reequilibrá-los. Além disso, o processo de formação de um Reikiano durava a vida inteira, por vezes um aluno demorava 10 anos para passar de um nível para o outro após longo período de prática e dedicação (USUI; PETTER, 1999), no ocidente isso ocorre quando a pessoa tem disponibilidade de tempo e dinheiro para pagar o curso do próximo nível, o que reflete a estrutura da cultura onde o tempo é escasso e tudo é transformado em produto.

Diversas linhas de Reiki foram criadas com sua proliferação pelo mundo, novas técnicas de aplicação, novos símbolos e outros conceitos foram incorporados a sua prática, sendo variados as formas e métodos de aplicação dessa técnica atualmente. No Brasil, dentre as PIC encontradas em pesquisa realizada pelo Ministério da Saúde em 2004, o Reiki foi identificado como a mais utilizada no Sistema Único de Saúde (SUS) (BRASIL, 2006). Isso pode ser atribuído a inúmeros motivos, dentre eles o fato de náo necessitar de procedimentos invasivos, ter raros registros de contraindicação, não necessitar de esforço físico e possibilitar uma prática de restabelecimento de energia de fácil e simples acesso. Nos Estados Unidos da América o National Center of Complementary and Alternative Medicine (NCCAM) caracteriza o Reiki como uma terapia complementar (NATIONAL..., 2011).

Socialmente o Reiki tem um papel importante no desenvolvimento da consciência do autocuidado, pois inicialmente para se aplicar Reiki em outras pessoas é necessário passar por um processo de purificação energética de 21 dias. Isso leva um praticante de Reiki a desenvolver essa consciência que acaba disseminando e contagiando todos ao seu redor, criando, assim, de maneira sutil, a consciência coletiva do autocuidado.

TT foi inventado pela enfermeira Dolores Krieger em 1972, e baseia-se no reequilíbrio e harmonizaçáo do CE do ser humano. Para os praticantes desta técnica o corpo é um sistema dinâmico de energia que interliga os aspectos físicos, emocionais, mentais e espirituais, e trabalhando no CE pode-se, consequentemente, interferir nestas diferentes dimensões facilitando assim a cura (GERBER, 2001).

Os passos para a realização do TT são: centralização: momento onde o terapeuta se centra na sensibilidade de suas mãos a fim de perceber o outro e curá-lo, utiliza-se de exercícios específicos para que o terapeuta atinja seu equilíbrio e silencie a mente; acesso $e$ avaliação do CE do paciente: o terapeuta toca o $\mathrm{CE}$ do paciente colocando suas mãos numa distância de 
6 a $12 \mathrm{~cm}$ da pele percorrendo-o no sentido crânio caudal percebendo qualquer desarmonia existente, como: pressão, tamanho e forma, diferenças de temperatura, formigamentos, choques elétricos e enrugamentos; tratamento e modulação do CE: depois que as alteraçôes são constatadas, o terapeuta deve restituir o padráo natural do CE através do alisamento, desobstrução e oposiçáo de sensações; balanceamento final e estabelecimento do fluxo energético: o terapeuta mantém o CE do paciente homogêneo e harmônico, fluindo em harmonia no sentido crânio caudal, direcionando a energia para a região das glândulas suprarrenais; avaliaçẫo: o terapeuta reavalia os achados no CE do paciente para comparar os achados com os da próxima sessão, nem sempre ele consegue uma harmonização total e é importante o terapeuta estar ciente de suas limitações. Além destes procedimentos é necessário que o terapeuta esteja saudável, centralizado e equilibrado do ponto de vista energético e com a intenção de ajudar o paciente (KRIEGER, 1975; MARTA, 1999; OLIVEIRA, 2000).

TC foi criado por Janet Mentgen, uma enfermeira que trabalhou 43 anos observando a conexão interrelacional entre enfermeiras e pacientes, para, após isso, criar Toque de cura como tratamento energético em 1989, a fim de expandir esta conexão. Trata-se de uma terapia energética que envolve o uso das mãos do praticante sobre e no corpo do paciente, utiliza do toque para acessar e determinar áreas de desequilíbrio de energia, percebidas como mudanças na temperatura, textura, ou vibração. $\mathrm{O}$ terapeuta desbloqueia a energia no corpo, promovendo cura física e equilíbrio emocional, mental e espiritual. $\mathrm{O}$ TC irá restaurar a harmonia e o equilíbrio do sistema de energia da pessoa para ajudá-la na "autopoiese" (POST-WHITE et al., 2003).

Essas técnicas se distinguem em vários aspectos, primeiramente o Reiki, ao que tudo indica, é uma técnica milenar que tem sua origem no oriente, utiliza de símbolos e sons sagrados para a canalização de energia vital, tem como premissa um processo de 21 dias de autoaplicaçáo para o iniciado poder aplicar em outras pessoas, sendo recomendado sempre que este se autoaplique para se manter saudável, porém, diferente do TT e do TC, náo necessita que o terapeuta esteja centrado e em paz, com a intenção de ajudar o paciente para ser aplicado.

O TT e o TC, diferentemente do Reiki, foram criados no ocidente recentemente, trabalham com o CE do ser humano, não utilizam de símbolos nem sons, apenas a percepção energética das mãos do terapeuta para harmonizar o CE do paciente, além $\mathrm{de}$, como foi citado anteriormente, necessitarem que o terapeuta esteja centrado e harmonizado com a intenção de ajudar o paciente. Todas essas técnicas de imposição de máos trabalham de alguma forma com a noção de autocuidado, o Reiki por necessitar de um processo de 21 dias de autoaplicação para poder ser aplicado em outras pessoas, estimulando, assim, que o terapeuta se cuide antes de poder cuidar do outro, o TT e o TC, por sua vez, trabalham com o autocuidado por proporcionarem exercícios de centramento e harmonização do terapeuta antes que ele cuide do outro.

Estudos realizados com essas práticas têm mostrado que o relaxamento é um dos efeitos mais comuns reportados. O estresse-ansiedade é caracterizado por um estado de tensão psicossomática que pode ser trabalhado através das técnicas de imposição de mãos por estas produzirem relaxamento e, diferente de fármacos, praticamente sem registros de contra indicação com baixo custo de aplicação (PETERS, 1999).

O estresse foi introduzido primariamente no campo da saúde em 1936, pelo fisiologista canadense Hans Selye, para designar a resposta geral e inespecífica do organismo a um estressor ou a uma situação estressante. É entendido como estresse o estado gerado por estímulos que acarretam em excitação emocional acionando o sistema nervoso autônomo simpático (SNAS), e um processo de adaptação que se caracteriza pelo aumento de secreção de adrenalina, noradrenalina e cortisol, gerando inúmeras manifestações sistêmicas, com alteraçóes psicológicas e fisiológicas (LABRADOR; CRESPO, 1994).

Diferentes respostas ao estresse podem ocorrer, variando de acordo com o indivíduo, sua percepção do estímulo, de fatores ambientais e genéticos, que determinam a resposta diante da situação estressora e a forma como o indivíduo será afetado. A ativação fisiológica frequente decorrente de estímulos estressores recorrentes pode levar ao aparecimento de distúrbios diversos, como, por exemplo, a ansiedade (MARGIS et al., 2003).

A ansiedade é uma experiência humana que preenche um papel funcional na interaçáo com o meio ambiente, mas também pode ocorrer como sintoma de várias doenças, sob a forma de estresse ou como distúrbio psiquiátrico. Pode ser definida como um estado emocional desagradável acompanhado de desconforto somático, sua ação fisiológica é semelhante a do estresse, ativação do SNAS e secreção dos hormônios citados. Está comumente relacionada a um evento futuro e, por vezes, considerada desproporcional a uma ameaça real (NIMA et al., 2013). 
A ansiedade e o estresse têm suas raízes em diversas reaçóes motivadas pela emoção de medo, do ponto de vista biológico em resposta aos perigos encontrados no meio ambiente, quando, por exemplo, um animal depara com uma ameaça ao seu bem-estar, à sua integridade física, ou até mesmo à sua sobrevivência. Em seu aspecto social, devido a inúmeros aspectos da vida moderna que geram reaçóes de preocupação, tensão e medo. Esses sintomas, de estresse e ansiedade, produzem, portanto, um estado de vigilância no organismo, a fim de que o indivíduo possa se manter alerta para enfrentar desafios naturais e/ou sociais (LABRADOR; CRESPO, 1994; MARGIS et al., 2003; NIMA et al., 2013).

Embora a ansiedade e o estresse sejam, portanto, reaçôes fisiológicas positivas de manutenção da vida e adaptação biológica, psicológica e social a diferentes estímulos do ambiente (MARGIS et al., 2003), essas respostas fisiológicas têm sido tratadas, principalmente, com medicamentos responsáveis por efeitos adversos (NIMA et al., 2013), no entanto, técnicas de imposição de mãos, como Reiki, Toque terapêutico e Toque de cura, podem atuar muito positivamente no controle da ansiedade e estresse, sem contraindicação e com baixo custo de aplicaçáo (THOMAS et al., 2013). O objetivo deste artigo é analisar sistematicamente a literatura sobre o uso e efeito dessas práticas integrativas e complementares no cuidado do estresse-ansiedade.

\section{Metodologia}

O método selecionado neste trabalho foi o da Revisão Sistemática da Literatura (RSL) pela necessidade de compilação das evidências científicas disponíveis, construindo assim conclusōes advindas de diferentes fontes (GONÇALO et al., 2012). Após a escolha do tema de interesse foram realizadas a busca e seleção dos artigos conforme consta na Figura 1. Posteriormente os estudos selecionados foram analisados criticamente, segundo o uso e efeito, objeto de estudo, metodologia, fator de impacto, país, ano, resultados e conclusões do estudo. Para avaliar o fator de impacto (FI) dos periódicos foi utilizado o índice do Journal Citation Reports (THOMSON..., 2013).

Foram adotados como critérios de inclusão: artigos de qualquer data e nacionalidade; artigos de língua inglesa ou portuguesa que continham em seu título ou resumo as palavras-chave: "Reiki and stress"; "Reiki andanxiety"; "Therapeutictouchand stress"; "Therapeutictouchandanxiety"; "Healingtouchand stress"; "Healingtouchandanxiety"; "Reiki e stress"; "Reiki e ansiedade"; "Toque terapêutico e stress";
"Toque terapêutico e ansiedade"; "Toque de cura e stress"; "Toque de cura e ansiedade".

Foram adotados como critérios de exclusão: artigos que relatem experimentos que não foram realizados em seres humanos; artigos que não eram sobre os sinais pesquisados; artigos indisponíveis na íntegra nas diferentes bases de dados; artigos que não relatavam o uso/efeito das técnicas de imposição de mãos no estresse-ansiedade; artigos que fossem duplicatas; artigos de revisão de literatura.

\section{Resultados}

A primeira publicação indexada sobre a aplicação das técnicas de imposição de mãos no estresse-ansiedade é do ano de 1999, o que revela um campo relativamente novo de pesquisa. Além disso, conforme as Tabelas 1 , 2 e 3 existe uma concentração de estudos (15 dos 24 artigos) nos últimos 6 anos, o que pode ser um indício do maior interesse da comunidade científica por essas técnicas no cuidado do estresse-ansiedade nos últimos anos.

Dos 24 artigos analisados, 14 foram publicados nos EUA. No Canadá, foram publicados 5 artigos, na Inglaterra 2, Irã, Brasil e Holanda publicaram 1, respectivamente. Os dados demonstram a predominância de estudos na América do Norte, 19, em sua maior parte nos EUA, 14, o que pode ser explicado pelo alto investimento que este país faz em pesquisas em PIC, sobretudo após a criação do NCCAM em 1998 (NATIONAL..., 2011).

Os estudos analisados apresentam uma predominância metodológica quantitativa, 21, destes 14 são estudos experimentais, dos quais 11 obtiveram resultados significativos e 3 resultados não significativos, 5 estudos utilizaram metodologia quase-experimental, dos quais 3 alcançaram resultados significativos e 2 resultados não significativos, e 2 fizeram uso da metodologia observacional, ambos com resultados significativos. Apenas 3 estudos utilizaram uma abordagem metodológica qualitativa, sendo 1 o relato de uma série de casos (MELAND, 2009) e os outros 2 estudos multimétodos (WILKINSON et al., 2002; RICHENSON et al., 2010). Um dado interessante revelado pelo estudo de Richenson et al. (2010) foi o desenvolvimento da consciência de autocuidado por parte dos participantes, esta variável só foi possível de ser constatada devido aos dados qualitativos, o que significa que a abordagem qualitativa pode revelar achados de pesquisa que não são demonstráveis por dados quantitativos. No estudo de Meland (2009) os participantes sentiram uma diminuiçáo da ansiedade após 3 semanas de aplicação do Reiki, além de se 


\section{Busca e seleção dos artigos}

Formulação da pergunta de pesquisa: Qual é o uso e o efeito das TIM em pessoas com stress-ansiedade?

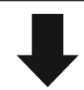

Busca realizada em outubro/2013 nas bases de dados: PubMed, Lilacs, Embase, Ebsco e Web OF Science com os descritores: "Reiki and stress", "Reiki and anxiety", "TT and stress", "TT and anxiety", "HT and stress", "HT and anxiety", "Reiki e stress", "Reiki e ansiedade", "Toque terapêtico e stress", "Toque terapêutico e ansiedade", "Toque de cura e stress", "Toque de cura e ansiedade".

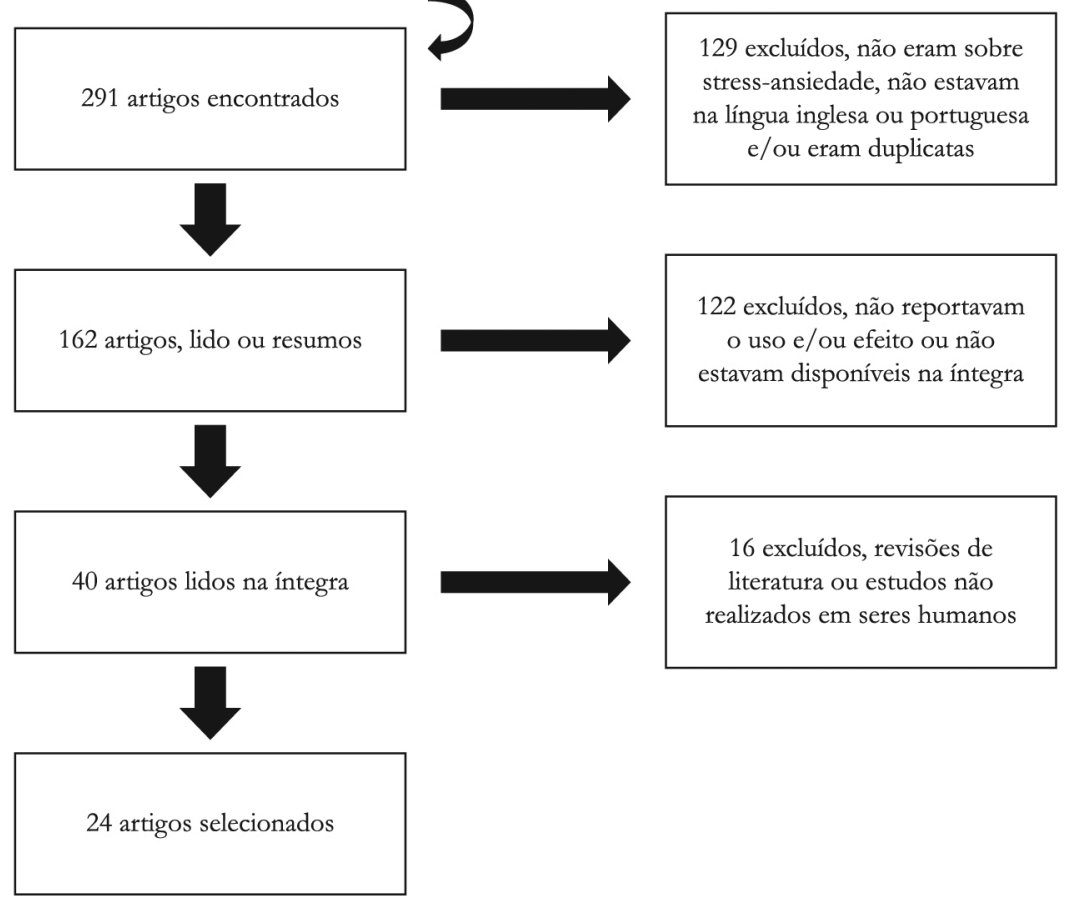

Figura 1. Busca e seleção dos artigos.

sentirem melhor, menos depressivos e mais relaxados. No estudo de Wilkinson et al. (2002), além dos resultados positivos das mensuraçôes quantitativas se observou nos resultados qualitativos que as pessoas relataram maior crescimento da saúde mental e emocional do que física e espiritual.

Os instrumentos utilizados nos estudos para medir os efeitos das técnicas de imposição de mãos no estresse-ansiedade foram diversos, como: aferimento de sinais vitais, como pressão sanguinea, batimento cardíaco, tensão muscular, amplitude do pulso, temperatura da pele, cortisol salivar e secreção de IgA (FRANK et al., 2007; ENGLE; GRANEY, 2000; BERGER; TAVARES; BERGER, 2013; ZOLFAGHARI; EYBPOOSH; HAZRATI, 2012;
WARDELL; ENGEBRETSON, 2001; BEARD et al., 2011; BOWDEN; GODDARD; GRUZELIER, 2010; BEARD et al., 2011); questionários de avaliação como State-Trait Anxiety Inventory (BEARD et al., 2011; LARDEN; PALMER; JANSSEN, 2004; LAFRENIERE et al., 1999; BOWDEN; GODDARD; GRUZELIER, 2011), Spielberger Anxiety Inventory (WARDELL; ENGEBRETSON, 2001; GOMES; SILVA; ARAÚJO, 2008), Anxiety and Stress Scale (BOWDEN; GODDARD; GRUZELIER, 2010; BUSCH et al., 2012), Hospital Anxiety and Stress Scale (BUSCH et al., 2012), Burn Specific Pain Anxiety Scale (REXILIUS et al., 2002), Profile of Mood States (BOWDEN; GODDARD; GRUZELIER, 2011), Beck Anxiety Inventory (MELAND, 2009), Mini Mental 
Tabela 1. Estudos do Reiki no estresse-ansiedade.

\begin{tabular}{|c|c|c|c|}
\hline Referência & $\begin{array}{c}\text { Técnica } \\
\text { NS/DI/GE }\end{array}$ & Uso & Efeito \\
\hline $\begin{array}{l}\text { Berger, Tavares e } \\
\text { Berger, } 2013\end{array}$ & $\begin{array}{l}\text { Reiki e TT NS- N/I } \\
\text { DI- N/I GE- } 31\end{array}$ & $\begin{array}{l}\text { Pacientes terminais } \\
\text { com ansiedade }\end{array}$ & $\begin{array}{l}\text { Melhora significativa, narrativas de } \\
\text { aumento do bem-estar }\end{array}$ \\
\hline $\begin{array}{l}\text { Marcus, O’Neill e } \\
\text { Kopar, } 2013\end{array}$ & $\begin{array}{l}\text { Reiki NS-N/I DI- } \\
282 \text { GE-145 }\end{array}$ & $\begin{array}{l}\text { Estresse e ansiedade em } \\
\text { pacientes com câncer }\end{array}$ & $\begin{array}{l}\text { Melhora significativa dos sintomas de } \\
\text { estresse e ansiedade }\end{array}$ \\
\hline $\begin{array}{l}\text { Bowden, Goddard e } \\
\text { Gruzelier, } 2011\end{array}$ & $\begin{array}{l}\text { Reiki NS-06/DI-30 } \\
\text { GE-20/GC-20 }\end{array}$ & $\begin{array}{l}\text { Bem-estar de estudantes } \\
\text { universitários }\end{array}$ & $\begin{array}{l}\text { Melhora significativa da ansiedade } \\
\text { após a quinta sessão }\end{array}$ \\
\hline Beard et al., 2011 & $\begin{array}{l}\text { Reiki NS-16/DI-50 } \\
\text { GE-36/GC-18 }\end{array}$ & $\begin{array}{l}\text { Homens com ansiedade } \\
\text { em radioterapia }\end{array}$ & Não houve diferença significativa \\
\hline $\begin{array}{l}\text { Bowden, Goddard e } \\
\text { Gruzelier, } 2010\end{array}$ & $\begin{array}{l}\text { Reiki NS-12/DI-20 } \\
\text { GE-18/GC-17 }\end{array}$ & $\begin{array}{l}\text { Bem-estar de estudantes } \\
\text { universitários }\end{array}$ & $\begin{array}{l}\text { O GE obteve uma redução significativa } \\
\text { do estresse-ansiedade comparado ao GC }\end{array}$ \\
\hline $\begin{array}{l}\text { Richenson et al., } \\
2010\end{array}$ & $\begin{array}{l}\text { Reiki NS-08/DI-45 } \\
\text { GE-13/GC-12 }\end{array}$ & Idosos com ansiedade & $\begin{array}{l}\text { Promoção de saúde, relaxamento e } \\
\text { consciência de autocuidado }\end{array}$ \\
\hline Meland, 2009 & $\begin{array}{l}\text { Reiki NS-08/DI-20/ } \\
\text { GE-06 }\end{array}$ & $\begin{array}{l}\text { Ansiedade em idosos } \\
\text { com demência }\end{array}$ & $\begin{array}{l}5 \text { dos } 6 \text { participantes experimentaram } \\
\text { diminuição da ansiedade depois da sessão }\end{array}$ \\
\hline Potter, 2007 & $\begin{array}{l}\text { Reiki NS-02/DI-54 } \\
\text { GE-17/GC-15 }\end{array}$ & $\begin{array}{l}\text { Ansiedade em mulheres } \\
\text { com câncer de mama }\end{array}$ & Não houve diferença significativa \\
\hline $\begin{array}{l}\text { Tsang, Carlson e } \\
\text { Olson, } 2007\end{array}$ & $\begin{array}{l}\text { Reiki NS-07/DI-45 } \\
\text { GE-08/GC-08 }\end{array}$ & $\begin{array}{l}\text { Ansiedade em pessoas } \\
\text { com câncer }\end{array}$ & Melhora significativa \\
\hline $\begin{array}{l}\text { Wardell e } \\
\text { Engebretson, } 2001\end{array}$ & $\begin{array}{l}\text { Reiki NS-01/DI-30 } \\
\text { GE-23 }\end{array}$ & $\begin{array}{l}\text { Pessoas com sintoma } \\
\text { de ansiedade }\end{array}$ & Melhora significativa \\
\hline
\end{tabular}

N/I - Não informado; NS - Número de sessões; DI - Duração da intervenção em minutos; GE - Grupo experimental; GC - Grupo controle; GP - Grupo placebo.

Tabela 2. Estudos DO TT no estresse-ansiedade.

\begin{tabular}{|c|c|c|c|}
\hline Referência & $\begin{array}{c}\text { Técnica } \\
\text { NS/DI/GE }\end{array}$ & Uso & Efeito \\
\hline Berger et al., 2013 & $\begin{array}{l}\text { TT e Reiki NS- N/I DI- } \\
\text { N/I GE- } 31\end{array}$ & $\begin{array}{l}\text { Pacientes terminais com } \\
\text { ansiedade }\end{array}$ & $\begin{array}{l}\text { Melhora significativa, } \\
\text { narrativas de aumento do } \\
\text { bem-estar }\end{array}$ \\
\hline Busch et al., 2012 & $\begin{array}{l}\text { TT NS-10/DI-10 GE- } \\
\text { 17/GP-22 }\end{array}$ & $\begin{array}{l}\text { Ansiedade em pessoas com } \\
\text { queimaduras }\end{array}$ & $\begin{array}{l}\text { A ansiedade pela dor diminuiu } \\
\text { significativamente na } 10^{\mathrm{a}} \text { sessão }\end{array}$ \\
\hline $\begin{array}{l}\text { Zolfaghari, Eybpoosh } \\
\text { e Hazrati, } 2012\end{array}$ & $\begin{array}{l}\text { TT NS-01/DI-10 GE- } \\
\text { 23/GC-23/GP-23 }\end{array}$ & $\begin{array}{l}\text { Ansiedade em mulheres } \\
\text { fazendo cateterização } \\
\text { cardíaca }\end{array}$ & Melhora significativa \\
\hline $\begin{array}{l}\text { Gomes, } \\
\text { Silva e Araújo, } 2008\end{array}$ & $\begin{array}{l}\text { TT NS-03/DI-N/I GE- } \\
21 / \mathrm{GP}-21\end{array}$ & $\begin{array}{l}\text { Ansiedade em estudantes } \\
\text { universitários }\end{array}$ & Melhora significativa \\
\hline Smith e Broida, 2007 & $\begin{array}{l}\text { TT NS-08/DI- 30/ } \\
\text { GE-24 }\end{array}$ & $\begin{array}{l}\text { Estresse dos terapeutas e } \\
\text { pacientes }\end{array}$ & $\begin{array}{l}\text { Diminuição do estresse por } \\
\text { curto período de tempo }\end{array}$ \\
\hline Frank et al., 2007 & $\begin{array}{l}\text { TT NS-01/DI-10/GE- } \\
42 / \mathrm{GP}-40\end{array}$ & $\begin{array}{l}\text { Ansiedade em mulheres } \\
\text { passando por biópsia }\end{array}$ & $\begin{array}{l}\text { Não houve diferença } \\
\text { significativa }\end{array}$ \\
\hline $\begin{array}{l}\text { Larden, Palmer e } \\
\text { Janssen, } 2004\end{array}$ & $\begin{array}{l}\text { TT NS-07/DI-20 GE- } \\
\text { 18/GC-18/GP-18 }\end{array}$ & $\begin{array}{l}\text { Ansiedade em mulheres } \\
\text { grávidas com dependência } \\
\text { química }\end{array}$ & $\begin{array}{l}\text { Decréscimo significativo } \\
\text { no GE nos dias } 1,2 \text { e } 3 \\
\text { comparado com GC e GP }\end{array}$ \\
\hline Engle e Graney, 2000 & $\begin{array}{l}\text { TT NS-01/DI-N/IGE- } \\
\text { 11/GP-11 }\end{array}$ & $\begin{array}{l}\text { Estresse em estudantes } \\
\text { universitários }\end{array}$ & $\begin{array}{l}\text { Não houve diferença } \\
\text { significativa }\end{array}$ \\
\hline Lin e Taylor, 1998 & $\begin{array}{l}\text { TT NS-03/DI-20 GE- } \\
\text { 30/ GP-30/ GC-30 }\end{array}$ & $\begin{array}{l}\text { Ansiedade em pessoas } \\
\text { idosas }\end{array}$ & Melhora significativa \\
\hline Lafreniere et al., 1999 & $\begin{array}{l}\text { TT NS-03/DI-N/I GE- } \\
\text { 22/GC-19 }\end{array}$ & $\begin{array}{l}\text { Ansiedade em pacientes } \\
\text { com câncer }\end{array}$ & Melhora significativa \\
\hline
\end{tabular}

N/I - Não informado; NS - Número de sessões; DI - Duração da intervenção em minutos; GE - Grupo experimental; GC - Grupo controle; GP - Grupo placebo. 
Tabela 3. Estudos do HT no estresse-ansiedade.

\begin{tabular}{|c|c|c|c|}
\hline Referência & $\begin{array}{c}\text { Técnica NS/DI/ } \\
\text { GE }\end{array}$ & Uso & Efeito \\
\hline Thomas et al., 2013 & $\begin{array}{l}\text { HT NS- } 04 \text { DI- } 30 \\
\text { GE- } 11 \text { GC }-06\end{array}$ & $\begin{array}{l}\text { Ansiedade e estresse em } \\
\text { pacientes hospitalizados }\end{array}$ & $\begin{array}{l}\text { Redução significativa apenas do } \\
\text { estresse na } 2^{\mathrm{a}} \text { e } 4^{\mathrm{a}} \text { sessão }\end{array}$ \\
\hline Tang et al., 2010 & $\begin{array}{l}\text { HT NS-02/ DI-900 } \\
\text { GE-26 }\end{array}$ & $\begin{array}{l}\text { Estresse em enfermeiras } \\
\text { líderes após serem treinadas } \\
\text { no HT }\end{array}$ & Melhora significativa \\
\hline Post-White et al., 2003 & $\begin{array}{l}\text { HT NS-04/DI-45 } \\
\text { GE-119/GP-45 }\end{array}$ & $\begin{array}{l}\text { Ansiedade em pessoas com } \\
\text { câncer }\end{array}$ & Não houve diferença significativa \\
\hline Wilkinson et al., 2002 & $\begin{array}{l}\text { HT NS-02/DI-30 } \\
\text { GE-22 }\end{array}$ & Pessoas com estresse & Melhora significativa \\
\hline Rexilius et al., 2002 & $\begin{array}{l}\text { HT NS-03/DI-30 } \\
\text { GE-23/GC-13 }\end{array}$ & $\begin{array}{l}\text { Ansiedade em pessoas com } \\
\text { câncer }\end{array}$ & Não houve diferença significativa \\
\hline
\end{tabular}

N/I - Não informado; NS - Número de sessões; DI - Duração da intervenção em minutos; GE - Grupo experimental; GC - Grupo controle; GP - Grupo placebo.

State Exam (POTTER, 2007), Self-Rating Anxiety Scale (TSANG; CARLSON; OLSON, 2007), Hamilton Anxiety Rating Scale (THOMAS et al., 2013), Hospital Anxiety and Depression Scale (WILKINSON et al., 2002), escala visual analógica (FRANK et al., 2007; ENGLE; GRANEY, 2000; RICHENSON et al., 2010, SMITH; BROIDA, 2007; TANG et al., 2010; MARCUS; O'NEILL; KOPAR, 2013), narrativas (RICHENSON et al., 2010; LIN; TAYLOR, 1998) e escala autorreferida (MARCUS; O’NEILL; KOPAR, 2013).

Nos resultados apresentados na Tabela 1 , dos 24 artigos 10 utilizaram o Reiki, sendo que 8 deles obtiveram resultados estatisticamente significativos e 2 não significativos. $\mathrm{Na}$ Tabela 2 , constituída de 10 artigos de TT aplicado ao cuidado de estresseansiedade, 8 obtiveram resultados estatisticamente significativos, 1 , resultado náo significativo, e 1, resultado negativo. $\mathrm{Na}$ Tabela 3 , foram apresentados os 5 estudos sobre o HT, desses 3 obtiveram resultados estatisticamente significativos e 2 não significativos. Sendo que o estudo de Berger, Tavares e Berger(2013) utilizou 2 técnicas de imposição de máos diferentes, Reiki e TT, e portanto se insere nas Tabelas 1 e 2. É importante notar que a maioria dos estudos apresenta um número de amostra baixo, o que pode ter influenciado os estudos com resultados estatisticamente não significativos.

O único estudo em que foi medida uma variável que aponta para uma direção prejudicial das técnicas de imposição de mãos foi o trabalho de Engle e Graney (2000) com o TT, desenvolvido nos EUA com estudantes universitários. Nesse estudo, o grupo que recebeu o TT experimentou redução do batimento cardíaco e amplitude de pulso, porém uma vasoconstrição, e não vasodilatação como era esperado. Contudo os autores alegam que a importância dessa variável é desconhecida.

Em relação ao FI dos jornais em que os artigos foram encontrados observa-se que apenas 8 dos 24 foram publicados em jornais com descriçáo do FI no Journal Citation Reports. Destes, em ordem decrescente conforme o FI, o primeiro estudo foi o de Bowden, Goddard e Gruzelier (2010) publicado no periódico Brain Research Buletim, com FI 2,81, seguido do artigo de Frank et al. (2007) publicado no periódico Pain Medicine, cujo FI é de 2,34. Logo após, se apresenta o trabalho de Busch et al. (2012) publicado no Patient Education and Counseling com FI de 2,30, seguido do estudo de Tsang, Carlson e Olson (2007) e Post-White et al. (2003), ambos publicados no Integrative Cancer Therapies com FI de 2,13. Em seguida temos o trabalho de Engle e Graney (2000), publicado no Journal of Nursing Scholarship com FI de 1,49, seguido do artigo de Berger, Tavares e Berger (2013), publicado no Journal of Palliative Medicine com FI de 0,78, e por último o artigo de Richenson et al. (2010), publicado no Research Gerontological Nursing com FI de 0,73.

A maioria dos artigos náo foi publicada em periódicos com descrição do FI e aqueles jornais que cotam FI são todos considerados de baixo impacto, o que pode ser interpretado como um indício da dificuldade dos autores em publicar pesquisas sobre técnicas de imposição de mãos especificamente e PIC em geral em revistas de maior visibilidade, relegando esse campo do conhecimento à margem da hegemonia biomédica.

Dos 24 artigos selecionados, 8 utilizaram as técnicas de imposição de mãos em pacientes com estresse-ansiedade em tratamento do câncer, 4 em estudantes universitários e $4 \mathrm{em}$ idosos. Desses estudos, 18 tiveram resultados estatisticamente 
significativos, 5 foram considerados não significativos e ocorreram, na sua totalidade, em pacientes com câncer, o único resultado negativo foi apresentado em estudantes universitários. Nos 8 estudos restantes as técnicas de imposição de mãos foram utilizadas em pessoas sem patologias associadas e com sintomas de estresse-ansiedade; 1 em pessoas com queimaduras; 1 em enfermeiras após serem treinadas no HT; 1 em mulheres com sintomas de estresse-ansiedade durante procedimento de cateterização cardíaca; 1 em terapeutas ao aplicar as técnicas de imposição de mãos; 1 em mulheres grávidas com dependência química e sintomas de estresse-ansiedade; e 1 em pacientes hospitalizados e sintomas de estresse-ansiedade.

\section{Discussão}

Os estudos analisados que utilizaram mais tempo de intervenção e maior número de sessóes obtiveram resultados mais significativos, em relaçáo àqueles com duração menor e pequeno número de intervenção, o que indica que, quanto maior o tempo de aplicação e número de sessões, melhores serão os resultados. Isso sugere que as técnicas de imposição de mãos têm efeito acumulativo, como mencionado no estudo de Lafreniere et al. (1999), sobre o TT, que conclui que os melhores resultados foram obtidos na última sessão.

Dos 24 estudos, 10 não relatam, em sua metodologia, o nível de experiência do terapeuta que aplicou a técnica de imposição de mão, sendo que 3 desses estudos tiveram resultados não significativos e 7 significativos. Isso pode constituir um viés metodológico importante nesse campo de pesquisa, já que, diferentemente de um fármaco, a efetividade dessas técnicas está diretamente relacionada à experiência do terapeuta que as administra. Dos 14 estudos voltados para essa questáo, 13 utilizaram terapeutas experientes (com 2 ou mais anos de experiência), dentre estes 11 obtiveram resultados significativos e 2 não significativos; o único estudo que relata experimento onde as técnicas foram administradas por terapeutas inexperientes os resultados não foram significativos. No estudo de Wilkinson et al. (2002), onde diversos praticantes de níveis diferentes aplicaram o HT, foi constatada uma melhora significativa maior dos níveis de $\operatorname{IgA}$ nos participantes que receberam tratamento de terapeutas mais experientes em relação aos que receberam tratamento de novatos.

Outro possível viés metodológico é em relação à padronização, nos estudos que apresentam o protocolo associado à aplicação da técnica, observa-se que a maioria deles obteve resultados não significativos com alguns poucos resultados significativos. Entretanto, nos estudos que utilizaram uma abordagem individual e não padronizada, todos tiveram resultados significativos, o que pode ser um indício de que os efeitos dessas técnicas são mais bem percebidos pelos pacientes e efetuados pelos terapeutas sob abordagem fenomenológica.

Este mesmo viés ocorre em relação à abordagem metodológica, a premissa de uma experiência ser estatisticamente significativa, ou não, polariza o conhecimento dificultando outras análises e a experiência individual dos participantes, gerando um viés que suscita a questão: "O que significa estatisticamente significativo ou não em uma experiência altamente subjetiva e individual?" Estudar técnicas como o Reiki, Toque terapêutico e Toque de cura apenas como se estuda a aplicação de um fármaco é uma forma de deturpar a lógica dessas técnicas terapêuticas, cada instrumento tem o seu lugar e seu modo apropriado de utilização e é imprescindível que isto seja enfatizado. Este modelo de pesquisa aumenta a polarização cultural na construção do que é um conhecimento legítimo, uma evidência epistemologicamente redutiva, marginalizando dados qualitativos e desenhos interpretativos que se alinham com os efeitos destas técnicas (BROOM; ADAMS, 2012).

É importante ressaltar que considerar o resultado de um estudo estatisticamente significativo ou não advém de uma construção bioestatística na qual se avalia a evidência contra o que se denomina hipótese nula, a evidência em oposto à hipótese nula é avaliada pelo valor de p, que significa a diferença probabilística entre os grupos estudados, quanto menor o p, maior a evidência contra a hipótese nula. A grande maioria dos ensaios clínicos considera a probabilidade menor do que $5 \%(\mathrm{p}<0,05)$ como limite para afirmar que o efeito observado é real, e náo obtido pelo acaso. Isso não significa que uma intervenção que obtém resultados estatisticamente não significativos não tenha efeitos, mas que as evidências contra a hipótese nula são fracas (COUTINHO; CUNHA, 2005).

Alguns estudos que chamam atençấo devido a sua abordagem metodológica e seus resultados são os trabalhos de Wardell e Engebretson (2001), de Bowden, Goddard e Gruzelier (2010) e de Wilkinson et al. (2002), nos quais foram estudados os marcadores biológicos para verificar o possível relaxamento proporcionado pelas técnicas de imposição de máos, nos 2 estudos em que foram mensurados os níveis de IgA salivar todos obtiveram resultados significativamente positivos (WARDELL; ENGEBRETSON, 2001; WILKINSON et al., 
2002), porém nos 2 estudos que mediram o nível de cortisol salivar os resultados não foram significativos (BOWDEN; GODDARD; GRUZELIER, 2010; WARDELL; ENGEBRETSON, 2001). O que levanta a pergunta se os marcadores biológicos são as melhores variáveis para verificar uma melhora no quadro de estresse-ansiedade, já que houve discordância de resultados entre os estudos com cortisol e IgA, além de que no estudo de Bowden, Goddard e Gruzelier (2010) e no de Wardell e Engebretson (2001) os resultados estatisticamente não significativos na mensuração do cortisol entraram em conflito com os resultados significativos obtidos pela utilização de outros instrumentos.

Perante a esta problemática, da adequação dos instrumentos aos objetos estudados, é importante ressaltar o trabalho de Zolfaghari, Eybpoosh e Hazrati (2012), o qual argumenta que o TT diminuiu significativamente a ansiedade mas não a tratou, $\mathrm{o}$ que pode ser atribuído à pequena quantidade de número de aplicaçôes da técnica, somente uma, e ao pequeno tempo de aplicação, de 10 a 15 minutos, porém suscita a reflexão de que caso essas técnicas sejam utilizadas como se utilizam medicamentos muito provavelmente elas não terão o efeito terapêutico proposto, mas funcionaram como paliativo, aliviando o sintoma e náo tratando a causa.

Considera-se a metodologia qualitativa a mais adequada para avaliar essas técnicas, a riqueza desses estudos está na descrição da experiência individual dos participantes, dado este impossível de ser mensurado, quantificado e analisado estatisticamente por estar na ordem da singularidade. A abordagem multimétodos pode enriquecer os trabalhos que avaliam esse tipo de prática por agregar o subjetivo e o objetivo, criando uma ponte entre os dados levantados, apontando contradiçóes e ampliando a visão científica a respeito das técnicas de imposição de mãos. O reducionismo quantitativo dificulta a criação de evidências, pois parte do pressuposto de uma verdade absoluta e uma única maneira de ver a mesma realidade, gerando marginalização de desenhos qualitativos pela comunidade científica, a padronizaçáo inerente dos desenhos de pesquisa controlados e randomizados não condiz com a lógica dessas técnicas (MOTTA; TAVARES, 2013).

Apenas 3 estudos utilizaram o cegamento simples de um dos grupos: no estudo de Bowden, Goddard e Gruzelier (2011) o cegamento foi utilizado de maneira que tanto o grupo controle quanto o grupo experimental recebessem relaxamento guiado através de sons da natureza. Em outro estudo de Bowden, Goddard e Gruzelier (2010) o cegamento foi realizado de forma que o grupo experimental náo recebesse o toque na aplicação de Reiki, mas apenas a imposição de mãos a uma distância de 3 a 30 polegadas de seu corpo, o grupo controle, por sua vez, não recebeu nada sendo que ambos participantes dos grupos estavam "cegos" durante a aplicação. No estudo de Lin e Taylor (1998) o cegamento foi realizado pela mímica realizada no grupo placebo, sendo que os participantes dos 2 grupos, experimental e placebo, náo sabiam o que estavam recebendo.

Dos 8 estudos que utilizaram grupo placebo, comparando com grupos expostos às técnicas de imposição de mãos, 5 tiveram resultados significativos e 3 não significativos. A pesquisa em PIC frequentemente recebe críticas que seus resultados provêm apenas de efeito placebo, por isso são enquadradas dentro do modelo de pesquisa biomédico de estudos cegos e duplos cegos, contudo isso se trata de um viés de pesquisa nessa área, pois a interação terapeuta-paciente é um componente crucial dos efeitos e resultados dessas práticas (LAFRENIERE et al., 1999). As técnicas de imposição de mãos por serem terapias que não utilizam de agulhas, medicamentos e nenhum outro arcabouço inerente ao imaginário do que é medicina na sociedade contemporânea, tem um efeito placebo muito mais brando comparadas a outras PIC como a acupuntura e a fitoterapia, que utilizam de instrumentos consagrados no imaginário biomédico como agulhas e fármacos.

A criação da PNPIC em 2006 é um reflexo desse paradigma em saúde, pois, apesar do Reiki ser a PIC mais utilizada no SUS, ele não entrou na política oficial, o que pode ser explicado pelo motivo desta técnica náo estar alinhada com a concepção de medicina da sociedade atual, e também pelo argumento de que náo há evidências, porém a questão do que consiste evidência é crucial neste campo de pesquisa. No entanto, mesmo no modelo mais ortodoxo de criação de evidências existem estudos clínicos desenvolvidos em âmbito nacional medindo os sistemas hematológicos e imunológicos de camundongos machos (OLIVEIRA, 2003) e aspectos psicofisiológicos e de qualidade de vida de idosos com sintomas de estresse (OLIVEIRA, 2013). Embora ambos os estudos tenham obtido resultados estatisticamente positivos, a comunidade científica continua com ressalvas contra essa técnica devido ao fato de não encontrar mecanismos "plausíveis" para seu funcionamento.

As técnicas de imposição de mãos são terapias que destoam da lógica da $\mathrm{MBE}$, esta obteve seu sucesso devido ao desenvolvimento da tecnologia que imbui valores particulares na natureza da produçáo do conhecimento. As técnicas de imposição de mãos são terapias que não necessitam de instrumentos 
tecnológicos por isso são marginalizadas por esta lógica, além de que seus efeitos são dificilmente captados por desenhos metodológicos considerados "padrão ouro", como estudos clínicos randomizados (BROOM; ADAMS, 2012).

Um reflexo deste modelo é o conteúdo dos artigos selecionados, os quais são pouco reflexivos, dedicando muito espaço para demonstrar resultados e pouco para discuti-los, o que gera uma ciência pouco reflexiva, que se foca apenas em números e estatísticas, não se indagando e refletindo o porquê desses números e o que eles representam (MOTTA; TAVARES, 2013). É necessário um discurso crítico da MBE onde o propósito não é desmantelar, mas contextualizar, equilibrar e enfatizar algumas vozes marginalizadas por esta lógica (BROOM; ADAMS, 2012).

Para que a pesquisa em técnicas de imposição de mãos se alinhe ao seu modelo de cuidado é necessário expandir o conceito de ciência, considerando-se que em sua base estão: observação, interpretação, conceitualização e teorização. Portanto, é necessário compreender que na pesquisa convencional o método científico é valorizado pelo seu rigor, porém esse mesmo rigor é válido para a abordagem das técnicas de imposição de mãos, de forma que as evidências não são rejeitadas, porém posicionadas em uma diferente visão de mundo (MOTTA; TAVARES, 2013).

Um limite deste trabalho é relativo ao pequeno número de artigos indexados identificados e analisados, que não torna possível fazer distinções em relaçáo às técnicas e seus efeitos. Assim, os resultados são oscilantes e discordantes, sendo os melhores obtidos em estudos que utilizaram uma abordagem metodológica qualitativa que está na ordem do subjetivo e individual, com o apoio de terapeutas experientes, maior número de sessóes e tempo de intervenção. No entanto, os méritos do trabalho alcançam o fato de que se trata de um campo novo e constituído de pesquisas de vanguarda para o cuidado de dois dos maiores sintomas da "epidemia de mal-estar” da sociedade mundial (LUZ, 2005).

\section{Conclusão}

O campo de pesquisa em PIC é relativamente novo e a pesquisa em técnicas de imposição de mãos, por sua vez, é mais nova ainda. Devido a isso, os resultados desta revisão são relativamente limitados, embora promovam um vislumbre dos possíveis efeitos dessas técnicas, assim como os vieses metodológicos provenientes de estudos quase exclusivamente quantitativos.
A complexidade da pesquisa em técnicas de imposição de mãos fica clara pela maneira que estas destoam da lógica biomédica em sua forma de cuidado. A interação terapeuta-paciente é um ponto crucial a ser explorado, bem como a aplicação fenomenológica dessas técnicas. Para uma melhor compreensão desse tema futuros estudos devem incluir mensuraçóes qualitativas que deem voz aos terapeutas e pacientes, para entender como eles experimentam essas técnicas, como elas podem colaborar para a melhora da qualidade de vida, bem-estar e outras variáveis indispensáveis a uma visão de saúde ampla e multidimensional.

As técnicas de imposição de mãos colaboram com a integralidade do cuidado ao tratarem do ser humano de maneira multidimensional, com uma abordagem centrada no paciente, considerando dimensôes que vão além do biológico, como, por exemplo, o campo de energia. Colaboram, também, com a pluralidade dos saberes ao ampliar o escopo de atuação terapêutica e incluir formas alternativas de tratamento que destoam da lógica biomédica hegemônica. Além disso, promovem saúde, pois permitem a manutençáo e melhora da qualidade de vida com técnicas náo invasivas de baixo custo e com pequeno registro de contraindicaçóes.

\section{Agradecimentos}

Ao Dr. Ricardo Monezi e à Dra. Pâmela Siegel pela revisão e discussão do artigo.

\section{Referências}

BABENKO, P. C. Reiki: um estudo localizado sobre terapias alternativas, ideologia e estilo de vida. 2004. $114 \mathrm{f}$. Dissertação (Mestrado em Ciências Sociais)-Universidade Federal de São Carlos, São Carlos, 2004.

BEARD, C. et al. Effects of complementary therapies on clinical outcomes in patients being treated with radiation therapy for prostate cancer. Cancer, Boston, v. 117, n. 1, p. 96-102, 2011.

BERGER, L.; TAVARES, M.; BERGER, B. A Canadian experience of integrating complementary therapy in a hospital palliative care unit. Journal of Palliative Medicine, Brisbane, v. 16, n. 10, p. 1294-1298, 2013. http://dx.doi. org/10.1089/jpm.2013.0295

BOWDEN, D.; GODDARD, L.; GRUZELIER, J. A randomized controlled single-blind trial of the effects of Reiki and positive imagery on well-being and salivary cortisol. Brain Research Buletin, Londres, v. 81, n. 1, p. 66-71, 2010.

BOWDEN, D.; GODDARD, L.; GRUZELIER, J. A radomised controlled single-blind trial of the efficacy of Reiki at benefitting mood and well-being. Evidence 
Based Complementary and Alternative Medicine, Londres, v. 20, p. 1-8, 2011.

BRASIL. Ministério da Saúde. Portaria no 971 de 03 de maio de 2006. Aprova a Política Nacional de Práticas Integrativas e Complementares - PNPIC. Diário Oficial da República Federativa do Brasil, Poder Executivo, Brasília, DF, 2006.

BROOM, A.; ADAMS, J. Evidence-Based Healthcare in Context: critical social science perspective. Inglaterra: Ashgate, 2012.

BUSCH, M. et al. The implementation and evaluation of therapeutic touch in burn patients: an instructive experience of conducting a scientific study within a non-academic nursing setting. Patient Education and Counseling, Holanda, v. 89, n. 3, p. 439-446, 2012.

COUTINHO, E. S. F.; CUNHA, G. M. Conceitos básicos de epidemiologia e estatística para a leitura de ensaios clínicos controlados. Revista Brasileira de Psiquiatria, Rio de Janeiro, v. 27, n. 2, p. 146-151, 2005.

ENGLE, V. F.; GRANEY, M. J. Biobehavioral effects of therapeutic touch. Journal of Nursing Scholarship, Memphis, v. 32, n. 3, p. 287-283, 2000. http://dx.doi. org/10.1111/j.1547-5069.2000.00287.x.

FRANK, L. S. et al. Does therapeutic touch ease the discomfort or distress of patients undergoing stereotactic core breast biopsy? A randomized clinical trial. Pain Medicine, Boston, v. 8, n. 5, p. 419-424, 2007.

GERBER, R. Um guia prático de medicina vibracional. Sáo Paulo: Cultrix, 2001.

GOMES, V. M.; SILVA, M. J. P.; ARAÚJO, E. A. C. Efeitos gradativos do toque terapêutico na redução da ansiedade de estudantes universitários. Revista Brasileira de Enfermagem, Brasília, v. 61, n. 6, p. 841-846, 2008.

GONÇALO, C. S. et al. Planejamento e execução de revisões sistemáticas da literatura. Brasília Médica, Brasília, v. 49, n. 2, p. 104-110, 2012.

KRIEGER, D. Therapeutic touch: the imprimatur of nursing. American Journal of Nursing, [S.1.], v. 35, n. 5, p. 784-787, 1975.

LABRADOR, F. J.; CRESPO, M. Evaluación conductual hoy:un enfoque para el cambio en psicologia clínica y de la salud. Madrid: Pirámide S.A, 1994.

LAFRENIERE, K. D. et al. Effects of therapeutic touch on biochemical and mood indicators in women. The Journal of Alternative and Complementary Medicine, Canadá, v. 5, n. 4, p. 367-370, 1999. http://dx.doi.org/10.1089/ acm.1999.5.367.

LARDEN, C. N.; PALMER, M. L.; JANSSEN, P. Efficacy of therapeutic touch in treating pregnant impatients who have a chemical dependecy. Journal of Holistic Nursing, Canadá, v. 22, n. 4, p. 320-332, 2004. http://dx.doi. org/10.1177/0898010104269242

LIN, Y. E.; TAYLOR, A. G. T. Effects of therapeutic touch in reducing pain and anxiety in an elderly population. Integrative Medicine, Charlottesville, v. 1, n. 4, p. 155-162, 1998.
LUZ, M. T. A crise da saúde e a crise da medicina no final do milênio. Revista Ciência e Saúde Coletiva, Rio de Janeiro, v. 15, p. 145-176, 2005. Suplemento.

MARCUS, D. A.; O'NEILL, B. B.; KOPAR, J. L. Symptomatic improvement reported after receiving reiki at a cancer infusion center.American Journal of Hospice and Palliative Care, Pittsburgh, v. 30, n. 2, p. 216-217, 2013. http://dx.doi.org/10.1177/1049909112469275.

MARGIS, R. et al. Relação entre estressores, estresse e ansiedade. Revista de Psiquiatria do Rio Grande do Sul, Porto Alegre, v. 25, n. 1, p. 65-74, 2003.

MARTA, I. E. R. Utilização do toque terapêutico no processo de cuidar de pessoas com dor crônica não-neoplásica. 1999. 195 f. Tese (Doutorado em Enfermagem)-Escola de Enfermagem de Ribeirão Preto, Ribeirão Preto, 1999.

MELAND, B. Effects of reiki on pain and anxiety in the elderly diagnosed with dementia: $\mathrm{d}$ serie of case reports. Alternative Therapy Health Medicine, Illinois, v. 15, n. 4, p. 56-57, 2009.

MOTTA, P. M. R.; TAVARES, M. M. Evidence healthcare in context: critical social science perspectives. Cadernos de Saúde Pública, Rio de Janeiro, v. 29, n. 12, p. 2563-2564, 2013.

NATIONAL CENTER OF COMPLEMENTARY AND ALTERNATIVE MEDICINE - NCCAM. National Institutes of Health. Estados Unidos da América: National Institutes of Health, 2011. Disponível em: <http://nccam. nih.gov/health/reiki/introduction.htm?nav=gsa $>$. Acesso em: 19 out. 2013.

NIMA, A. A. et al. Anxiety, affect, self-esteem, and stress: mediation and moderation effects on depression. Plos One, San Francisco, v. 8, n. 9, p. e73265, 2013

OLIVEIRA, R. M. J.Avaliação de efeitos da prática de imposição de máos sobre os sistemas hematológicos e imunológico de comundongos macho. 2003. 96 f. Dissertação (Mestrado em Ciências)-Universidade de São Paulo, São Paulo, 2003.

OLIVEIRA, R. M. J. Efeitos da prática do Reiki sobre aspectos psicofisiológicos e de qualidade de vida de idosos com sintomas de estresse: estudo placebo e randomizado. 2013. 191 f. Tese (Doutorado em Ciências)-Universidade Federal de São Paulo, São Paulo, 2013.

OLIVEIRA, V. L. M. O toque terapêutico no alivio da dor e seu desconforto em doentes vasculares amputados com enfermidades arteriais de membros inferiores. 2000. $103 \mathrm{f}$. Dissertação (Mestrado em Enfermagem)-Universidade Guarulhos, Guarulhos, 2000.

PETERS, R. M. The effectiveness of therapeutic touch: a meta-analytic review. Nursing Science Quarterly, [S.1.], v. 12, n. 1, p. 52-61, 1999.

POST-WHITE, J. et al. Therapeutic massage and healing touch improve symptons in cancer. Integrative Cancer Therapies, Minneapolis, v. 2, n. 4, p.322-344, 2003.

POTTER, P. J. Breast biopsy and distress: feasibility of testing a reiki intervention. Journal of Holistic Nursing, Washington, v. 25, n. 4, p. 238-248, 2007. http://dx.doi. org/10.1177/0898010107301618. 
REXILIUS, S. J. et al. Therapeutic effects of massage therapy and healing touch on caregivers of patiente undergoing autologous hematopoietic stem cell transplant. Oncology Nursing Forum, Omaha, v. 29, n. 3, p. 35-44, 2002.

RICHENSON, N. E. et al. Effects of reiki on anxiety, depression, pain, and physiological factors in communitydwelling older adults. Research in Gerontological Nursing, Maine, v. 3, n. 3, p. 187-199, 2010.

SMITH, D. W.; BROIDA, J. P. Pandimensional filed pattern changes in healers and healess: experiencing therapeutic touch. Journal of Holistic Nursing, [S.1.], v. 25, n. 4, p. 226-227, 2007.

STEWART, J. C. The Reiki Touch: the essential handbook. Houston: The Reiki Touch, 1995.

TANG, R. et al. Improving the Well-Being of Nursing leaders through healing touch training. Journal of Alternative and Complementary Medicine, Winston-Salem, v. 16 , n. 8 , p. $837-841,2010$. http://dx.doi.org/10.1089/ acm.2009.0558.

THOMAS, L. S. et al. A pilot study: the effect of healing touch on anxiety, stress, pain, pain medication usage, and pain episode physiological measures in hospitalized sickle cell disease adults experiencing a vaso-occlusive. Journal of Holistic Nursing, Carolina do Norte, v. 20, n. 10, p. 1-14, 2013.

THOMSON REUTERS. Journal Citation Reports. EUA, 2013. Disponível em: <http://thomsonreuters.com/ journal-citation-reports/>. Acesso em: 20 de out. 2013.
TSANG, K. L.; CARLSON, L. E.; OLSON, K. Pilot crossover Trial of Reiki versus rest for treating câncerrelated fatigue. Integrative Cancer Therapies, Canada, v. 25 , n. 6, p. 25-35, 2007.

USUI, M.; PETTER, F. A. Manual de Reiki do Dr. Mikao Usui. Sáo Paulo: Pensamento, 1999.

VANDERVAART, S. et al. A systematic review of the therapeutic effects of reiki. Journal of Alternative and Complementary Medicine, Toronto, v. 15, n. 11, p. $1157-$ 1169, 2009. http://dx.doi.org/10.1089/acm.2009.0036.

VANDERVAART, S. et al. The effect of distant reiki on pain in women after elective Caesarean section: a double-blinded randomized controlled trial. BMJ Open, Toronto, v. 1, n. 1, p. 1-9, 2011.

WARDELL, D. W.; ENGEBRETSON, J. Biological correlates of Reiki Touch healing. Journal of Advanced Nursing, Houston, v. 33, n. 4, p. 439-445, 2001. http:// dx.doi.org/10.1046/j.1365-2648.2001.01691.x.

WILKINSON, D. S. et al. The clinical effectiveness of Healing Touch. Journal of Alternative and Complementary Medicine, Nashville, v. 8, n. 1, p. 33-47, 2002. http:// dx.doi.org/10.1089/107555302753507168.

ZOLFAGHARI, M.; EYBPOOSH, S.; HAZRATI, M. Effects of Therapeutic Touch on anxiety, vital signs, and cardiac dysrhythmia in a sample of iranian women undergoing cardiac catheterization: a quasi-experimental study. Journal of Holistic Nursing, Tehran, v. 30, n. 4, p. 225-234, 2012. http://dx.doi.org/10.1177/0898010112453325.

\section{Contribuição dos Autores}

Ambos autores foram responsáveis por todas as fases da pesquisa e elaboração do texto, sendo o segundo autor o orientador do trabalho original. 